\title{
Archéopages
}

Archéopages

Archéologie et société

40 | 04-07/2014

Villages

\section{La notion de village en France au premier Moyen Âge. Retour sur un débat}

\section{Édith Peytremann}

\section{(2) OpenEdition}

1 Journals

Édition électronique

URL : https://journals.openedition.org/archeopages/618

DOI : 10.4000/archeopages. 618

ISSN : 2269-9872

\section{Éditeur}

INRAP - Institut national de recherches archéologiques préventives

\section{Édition imprimée}

Date de publication : 1 juin 2015

Pagination : 84-91

ISSN : 1622-8545

\section{Référence électronique}

Édith Peytremann, «La notion de village en France au premier Moyen Âge. Retour sur un débat »,

Archéopages [En ligne], 40 | 04-07/2014, mis en ligne le 01 juillet 2016, consulté le 21 janvier 2022

URL : http://journals.openedition.org/archeopages/618; DOI : https://doi.org/10.4000/archeopages. 618

(c) Inrap 


\title{
La notion de village en France au premier Moyen Âge Retour sur un débat
}

\author{
Édith Peytremann Irrap
}

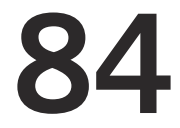

1. Villages et villageois au Moyen Âge, Actes du XXXI Congrès de la Société des historiens médiévistes de lenseignement supérieur public, Caen, 199o, Paris, Publications de la Sorbonne (coll. Histoire ancienne et médiévale, 26), $214 \mathrm{p}$.
Le village médiéval est une entité incontournable du récit historique. Elle est profondément ancrée dans notre imaginaire collectif, avec sa place, son église et parfois son château, auxquels il faut ajouter, à partir du XIX ${ }^{\mathrm{e}}$ siècle, sa mairie et son école. Cette représentation collective archétypale illustre parfaitement la complexité de l'objet village et apporte également des éléments d'explication au débat qu'il a suscité et qu'il suscite encore au sein de la communauté des médiévistes. Car si le village médiéval est reconnu, voire sublimé, il n'en va pas de même pour les cinq ou six siècles qui précèdent les $\mathrm{XI}^{\mathrm{e}}-\mathrm{XII}^{\mathrm{e}}$ siècles. Avant de s'interroger sur ce qu'est le village altomédiéval, il convient de revenir brièvement sur ce débat qui est aussi symptomatique du développement de l'archéologie médiévale et de la difficulté qu'éprouvent les médiévistes à intégrer à leur discours la documentation archéologique.

\section{Les fondements d'un débat}

Avant les années 1980, l'usage du mot «village» pour les périodes antérieures aux $\mathrm{XI}^{\mathrm{e}}-\mathrm{XII}^{\mathrm{e}}$ siècles ne pose pas spécialement de problème, même si les réalités qu'il recouvre sont variables selon les auteurs [ill.1] et s'orientent vers des domaines aussi bien juridique, géographique que social. C'est à partir des années 1980 et particulièrement depuis la parution des travaux de Robert Fossier (Chapelot, Fossier, 1980 ; Fossier, 1982) que l'emploi du terme «village » devient problématique, notamment pour les archéologues médiévistes. Robert Fossier, suivi par un certain nombre d'historiens et d'archéologues, réserve en effet l'emploi de ce mot pour désigner un regroupement compact de maisons fixes, associé à la présence de noyaux de rassemblement comme le cimetière, l'église ou le château, à une organisation du terroir environnant et à une prise de conscience communautaire. Les autres regroupements, que laissent entrevoir l'archéologie notamment, sont a contrario désignés comme des «habitats ruraux», dans la mesure où ils sont majoritairement construits en matériaux périssables, ne sont pas estimés fixes et ne comportent pas toujours un cimetière, une église ou un château. À la suite de ces thèses, plusieurs voix se sont fait entendre pour les contester partiellement ou entièrement, notamment chez les archéologues qui pouvaient s'appuyer sur les données d'une archéologie préventive débutante (Périn, 1992 ; Lorren, Périn, 1995). On retrouve un écho de ce débat dans la publication des actes du congrès de la Société des historiens médiévistes qui s'est tenu en 1990 ${ }^{\mathbf{1}}$. L'historien Adriaan Verhulst (1992) distingue deux approches différentes: l'une matérielle et géographique, réalisée par les archéologues; l'autre abordée sous l'angle de la communauté rurale, développée par les historiens. Prudent, il admet que l'existence du village est probable dans son sens matériel, dès La Tène et notamment à la période mérovingienne. En revanche, il reste sceptique sur le critère de la stabilité du fait du caractère « ambulant » ou éphémère du village du haut Moyen Âge, démontré par l'archéologie. Il conteste néanmoins l'argumentation de Robert Fossier en lui opposant les travaux d'historiens allemands ou belges. Ce dernier (Fossier, 1992) réaffirme avec force, en conclusion des actes, sa vision du village, quitte à user du néologisme de «protovillage» pour certains groupements de maisons possédant un ou plusieurs noyaux de rassemblement. Il insiste plus particulièrement sur «l'état d'esprit villageois». Dans le prolongement 
de ces débats ${ }^{2}$, paraît, sous la plume d'Élisabeth Zadora-Rio (1995), un important article qui tente de faire le point entre arguments textuels et arguments archéologiques en insistant sur trois aspects particuliers. Les deux premiers, plus géographiques, touchent à l'aspect quantitatif - combien de maisons pour faire un village? et, à la suite des travaux sur la ville, aux fonctions villageoises. Le troisième, aux dimensions plus historiques, concerne la communauté. Les cinq fonctions (funéraire, religieuse, économique, défensive et administrative) retenues par l'auteur reprennent de fait la définition de Robert Fossier. L'auteur, plus modéré que ce dernier - tout en reconnaissant à quelques découvertes archéologiques un certain nombre de critères villageois - conteste une origine du village à l'époque mérovingienne, s'appuyant pour cela sur la conception différente de l'espace à partir $\mathrm{du} \mathrm{XI} \mathrm{I}^{\mathrm{e}}$ siècle. Et c'est bien sur un problème de chronologie, mais pas uniquement, que repose ce débat. Des traces de celui-ci sont encore perceptibles dans le Dictionnaire du Moyen Âge (Gauvard, de Libera, Zinc, 2004). L'article «village » reprend dans ses grandes lignes les thèses de Robert Fossier, notamment sur la construction en dur et l'importance de la communauté. Il insiste sur la complexité du processus et pose la question «du seuil minimal de conditions que doit remplir un habitat donné pour être considéré comme un village » (Cursente, 2004, p. 1449). À son tour, il reconnaît l'existence d'habitats ruraux, produits «de phénomènes de densification et de coagulation $»^{3}$, mais leur conteste, pour la plupart d'entre eux, l'appellation de «village». Le site de Mondeville (Calvados) fait ainsi exception. Le premier Moyen Âge correspond donc à une «longue phase de croissance préparatoire» préalable à la «naissance massive des villages » liée «à la généralisation de noyaux de condensation que furent le cimetière, l'église, le château [...]». L'année 2003 est caractérisée par la parution de plusieurs publications archéologiques ayant en commun une déconstruction plus ou moins poussée des thèses de Robert Fossier. La portée de la dynamique villageoise et l'importance qu'il y a à définir l'objet «village » selon son contexte chronologique ont été soulignées à l'occasion d'un travail de thèse (Peytremann, 2003, p. 101). Nombre de critères avancés pour définir le «village» n'ont en effet qu'une validité chronologique restreinte liée à des phénomènes politiques et sociétaux. C'est également dans cette optique que se place Claude Raynaud quand il plaide «à propos du village, sur la nécessité de ne pas enfermer une forme de peuplement dans une région ou une période [...]» (Raynaud, 2003, p. 352-353). Dans un dossier des Nouvelles de l'archéologie consacré à l'habitat rural, Élisabeth Zadora-Rio revient sur les modèles établis et la pesanteur des paradigmes face aux données nouvelles fournies par l'archéologie. Elle montre que les modèles historiques, notamment celui du regroupement autour de l'église et du château, n'ont pas ou peu de réalité archéologique. Sans pour autant renier la thèse de l'encellulement avancée par Robert Fossier, elle propose alors une lecture fondée sur deux modèles, l'un à l'échelle macrohistorique concernant principalement les élites, l'autre à l'échelle microhistorique, relatif au site, le premier ne pouvant avoir que peu d'influence sur l'habitat. La dernière publication est un court article épistémologique qui reprend le débat exposé ci-dessus et plaide, afin d'en sortir, pour un changement d'objet «du village au réseau » en s'appuyant sur les travaux de Claude Raynaud et en développant une approche archéogéographique (Watteaux, 2003, p. 314). Ces publications indiquent un tournant, où il apparaît que l'intérêt de la recherche consiste plus à comprendre, sur une longue période, les transformations des modalités de peuplement et à en saisir les acteurs qu'à s'attarder sur des questions sémantiques. La focalisation de la problématique du village sur une définition a en effet sclérosé le débat en polémique stérile. De fait, il n'y a eu que peu d'études sur la question après 2003. Il est néanmoins impossible de ne pas mentionner les travaux de Jacques Morsel qui aborde la problématique sans qu'elle soit au centre de ses recherches. C'est en effet en s'interrogeant sur la spatialisation sociale et notamment sur celle des communautés d'habitants qu'il aborde «le village et l'habitat» (Morsel, 2007). À la suite d'autres médiévistes, il distingue nettement dans une logique sociologique le «village social», la communauté des habitants du «village physique», l'agglomération des habitants, en précisant que la spécificité de la communauté des habitants est d'être «définie par rapport à un espace commun ». Il précise bien que les agglomérations que l'on rencontre antérieurement ( $\mathrm{au} \mathrm{XI}{ }^{\mathrm{e}}$ siècle) n'étaient qu'une juxtaposition de cultivateurs dépendant de maîtres différents et sans relations entre eux, notamment par les liens du mariage (Morsel, 2007, p. 152). Cette approche s'inscrit à l'évidence dans la lignée des thèses de Robert Fossier, en faisant du Xi ${ }^{\mathrm{e}}$ siècle le siècle de la rupture. Mais que penser, quand, dans la suite de son discours, Jacques Morsel tente de démontrer que la relation sociale fondamentale pour la communauté d'habitants, c'est celle d'habiter. Corrigeant la thèse de Fossier sur la transformation de l'habitat au village, il affirme que « l'encellulement est, plus que la phase de formation des villages, celle de la formation de l'habitat proprement dit: avant, il n'existe aucun habitat, ni groupé ni dispersé, mais un autre rapport spatial qu'il conviendrait de désigner autrement» (Morsel, 2007, p. 154, note 180). La situation est devenue ubuesque pour les médiévistes travaillant plus particulièrement sur la campagne au premier Moyen Âge, puisque les termes d' «habitat» et de «village» désignant une forme de peuplement sont désormais 
1. Tableau récapitulatif des principales définitions du village dans la littérature historique et archéologique.

\section{\begin{tabular}{l|l} 
Auteurs & Définition (période chronologique de validité)
\end{tabular}}

Bloch M., 1931, p. 163

«[...] collectivité au même titre que la seigneurie. Le village et son finage sont l'œuvre d'un même groupe [...]; les manses sont les parts attribuées [...] à des sous-groupes plus petits. » (vIII $-x^{e} s$.)

\section{Roupnel G.,}

1931, p. 64

«[...], le village n'existe qu'en fonction des chemins de sa campagne.

Leur réunion lui donne sa raison d'être. Leur rencontre lui donne sa structure.»

\section{Fournier G., 1962, p. 260 et 311}

«[...] villages formés par la juxtaposition des manses.» et «petite localité rurale formée de la réunion des bâtiments et des terres qui appartenaient à des propriétaires plus ou moins riches ». ( $\mid x^{\mathrm{e}}-\mathrm{x}^{\mathrm{e}} \mathrm{s}$.)

\section{Duby G., \\ 1962, p. 65}

«[...] l'agglomération est constituée d'une juxtaposition de parcelles. » $\left(\mid x^{\mathrm{e}}-\mathrm{X}^{\mathrm{e}} \mathrm{s}.\right)$

\section{Salin É., \\ 1965, p. 422}

«[...] un rassemblement de paysans ayant des intérêts communs. » $\left(\mathrm{vl} \mathrm{e}^{\mathrm{e}} \mathrm{vI} \mathrm{I}^{\mathrm{e}} \mathrm{s}.\right)$
"Le village est une localité de petite taille, au plan lâche, occupée par des petits propriétaires d'exploitations familiales. » ( $\mathrm{VI} \mathrm{e}^{\mathrm{e}}-\mathrm{VII}{ }^{\mathrm{e}} \mathrm{s}$.)

\section{Chapelot, Fossier R., 1980, p. 133-134}

Pesez J.-M., 1981, p. 345
Bourin M., Durand R., 1984 p. 19
Le village achevé est caractérisé par «l'église, le château et le resserrement des maisons dans un site au plan organisé autour de ceux-ci qui forment avec la place, et dans certains cas l'enceinte, autant de pôles particuliers ». (à partir du xII $\mathrm{s}$.)

«[...] une entité, somme toute complexe, associant un bâti, habitat permanent fixé sur un site, un territoire agricole, le finage, et un groupe humain, doté d'une personnalité morale s'exprimant à travers diverses institutions et d'abord la paroisse et la communauté rurale. » (vers le $\mathrm{xIII}{ }^{\mathrm{e}} \mathrm{s}$.)

"Le village c'est donc, tout simplement et d'abord, un certain cadre monumental qui exprime les relations sociales des villageois et la nature de leurs solidarités. » 
Geary P. J., 1989, p. 132
«[...] le village est le centre de la vie religieuse et sociale. C'est un habitat groupé parfois fort éloigné des terres. C'est également une entité importante de la fiscalité. »( $v^{e}-\left.v\right|^{e} s$. $)$
Verhulst A., 1992, p. 10
«[...] un ensemble fonctionnel, transcendant la simple juxtaposition géographique de quelques fermes et maisons, aussi peu nombreuses qu'elles soient - un ensemble dont la fonctionnalité se manifeste dans des éléments communautaires comme des parcs de rassemblement pour le bétail, tels les trieux en Belgique, ou des communaux ou encore l'église et le cimetière. » (sens géographique et matériel)
Fossier R., 1995, p. 8
«Il faut réserver le mot, certes, à un groupement compact de maisons fixes, mais aussi à la présence de divers noyaux de rassemblement où les morts joueront le premier rôle, à une organisation cohérente du terroir environnant et, surtout, à l'apparition d'une prise de conscience communautaire sans laquelle il n'y a pas de "villageois", mais des habitants. "
Lorren C., cité dans Nissen-Jaubert A., 1996, p. 19

Zadora-Rio É., 1995, p. 147
«Groupe d'hommes qui se reconnaissent solidaires sur un finage dont ils ont conscience des limites. »
Nissen-Jaubert A., 1996, p. 17

\section{Cursente B.,} 2004, p. $1448-1450$
«[...] l'existence d'une communauté rurale ayant une personnalité juridique et celle d'un territoire agraire organisé, aux limites connues; [...] le regroupement durable, en un même lieu, d'un certain nombre de fonctions : religieuse, funéraire, défensive, administrative et économique - sans que l'éventail complet soit nécessairement représenté. » 
proscrits! On le voit, même si le débat a perdu de sa vigueur, il reste présent et certains médiévistes continuent de considérer la vie à la campagne durant les premiers siècles du Moyen Âge de manière étonnante, refusant aux paysans une vie sociale en dehors d'une structure foncière et d'un mode de faire-valoir. Au-delà de cette prise de position postmoderne, il convient, à partir des données réunies depuis trente ans que dure ce débat, de reprendre le dossier à la lumière du renouvellement des données archéologiques.

\section{Les critères utilisés pour parler du village}

Létymologie du terme village renvoie au mot villagium, groupe d'habitations rurales, dont la plus ancienne mention connue date de 1235 (Du Cange et al., 1883-1887, t. 8, coll. 330c). Aussi, en partant du postulat qu'il existe une forme de peuplement villageois au premier Moyen Âge, l'ensemble des critères qui font consensus ou qui sont discutables sont examinés à l'aide d'exemples archéologiques afin d'esquisser les grands traits du village du premier Moyen Âge.

À la lecture des principales définitions ${ }^{4}$ existantes, il ressort que le village renvoie à un groupe d'habitations assez important pour avoir une vie propre. Deux éléments apparaissent, l'un renvoyant au quantitatif, l'autre à la topographie. Ces deux notions renferment une part de subjectivité évidente. À partir de combien d'habitations considère-t-on en effet l'importance du groupe au premier Moyen Âge? Déjà en 1975, Guy Fourquin estimait que la taille du village mérovingien devait être plus proche du hameau, selon nos critères contemporains (Fourquin, 1975, p. 310-311). À titre d'exemple, le village de Laucheim, en Allemagne, comprend au maximum une dizaine de fermes pour la longue période qui s'étend du $\mathrm{VI}^{\mathrm{e}}$ au XII ${ }^{\mathrm{e}}$ siècle (Stork, 1997). Le village de Kircheim, en Bavière, fouillé sur une superficie légèrement inférieure à 1 ha, comprend au moins une dizaine d'habitations pour la période comprise entre le milieu du $\mathrm{v}^{\mathrm{e}}$ siècle et le milieu du VIII ${ }^{\mathrm{e}}$ siècle (Dannheimer, 1973; Christlein, 1980). C'est au moins sept unités agricoles qui sont recensées pour le village de Serris (Seine-et-Marne) aux VII ${ }^{\mathrm{e}}$-VIII ${ }^{\mathrm{e}}$ siècles sur une surface étudiée de 18 ha, plus si l'on y associe les découvertes effectuées sur la commune de Jossigny, à moins de $300 \mathrm{~m}$ de la limite sud du site de Serris (Gentili, 2010). C'est aussi une dizaine d'habitations qui est recensée sur le site du village de Saint-Gilles-deMissignac à Aymargues (Gard), fouillé sur environ 1,5 ha ${ }^{5}$. Franck Gérard utilise le terme de «hameau» pour désigner l'occupation des $\mathrm{VIII}^{\mathrm{e}}-\mathrm{IX}^{\mathrm{e}}$ siècles $\mathrm{du}$ site de Vallange (Moselle) qui regroupe six unités d'habitation, alors que le «village » qui lui succède en comprend au moins sept. La superficie étudiée est de 3,25 ha (Gérard, 2012, p. 39).

La topographie touche au groupement qui peut être, selon les cas et les périodes, plus ou moins lâche ou resserré. Un phénomène de concentration est observable à partir du milieu du viI ${ }^{\mathrm{e}}$ siècle (Peytremann, 2003, p. 324). Parmi les critères qui font consensus se trouve celui de la fonction économique principalement orientée vers l'activité agricole, attestée aussi bien par le mobilier carpologique, ostéologique et métallique que par les structures de stockage. Selon les cas peuvent s'ajouter des activités artisanales ou d'exploitation de matière première. Les villages de Vert-SaintDenis « Les Fourneaux » (Seine-et-Marne), de Saran (Loiret) ou de Villeroy (Yonne) peuvent être cités à titre d'exemple .

$\mathrm{La}$ «fonction » funéraire du village est plus discutable dans le sens où elle obéit à des développements chronologiques et sociologiques particuliers, dont la principale rupture est l'inscription des aires funéraires au sein de l'espace bâti à partir de la fin du viI ${ }^{\mathrm{e}}$ siècle. Auparavant, le cimetière se trouve à distance de l'espace bâti, dans un rayon de $2 \mathrm{~km}$ maximum. Du point de vue archéologique, la principale difficulté pour s'assurer d'une présence funéraire tient aux limites de fouilles. Des exemples nombreux de villages du premier Moyen Âge attestent néanmoins une aire funéraire collective et/ou des sépultures dispersées ou isolées. Parmi les plus connus possédant un cimetière peuvent être mentionnés les villages de Mondeville (Calvados), de Tournedos-sur-Seine (Eure), de Rigny-Ussé (Indre-et-Loire), de Saleux (Somme), de Sarrixing à Imling (Moselle), de la Grande-Paroisse, de Villiers-le-Sec ou de Serris (Seine-et-Marne) ${ }^{7}$. Les découvertes récentes réalisées à Aymargues (Gard) renforcent l'hypothèse du rapprochement des morts avec les vivants. Parmi les villages dont seules des sépultures dispersées sont connues, les cas de Genlis (Côte-d'Or) (Catteddu, 1992) ou de Saran (Loiret) (Jesset, à paraître) peuvent être mentionnés.

La fonction religieuse parfois avancée pour définir le village est, elle aussi, entachée d'une dimension chronologique. La grande majorité des bâtiments religieux connus prennent place au sein de l'espace bâti, là encore à partir du milieu du $\mathrm{VII}^{\mathrm{e}}$ siècle. Par ailleurs, certains correspondent à des bâtiments chrétiens à fonction funéraire. Les villages précédemment évoqués sont tous équipés d'un bâtiment religieux, dont la fonction cultuelle ou funéraire n'est pas toujours identifiée. D'autres sites, comme Airvault (Deux-Sèvres) ou Poigny (Seine-et-Marne) ${ }^{8}$, témoignent de la présence d'un édifice religieux, mais la faiblesse de la superficie explorée ne permet pas de les identifier avec certitude à un village.

La fonction administrative suggérée par certains auteurs apparaît anachronique pour les périodes concernées. Quant à la fonction défensive, elle semble discutable tant par son aspect chronologique que fonctionnel. Elle ne peut en aucun cas être généralisée. Avec ces deux fonctions, on touche ici aux limites des définitions entre la ville et le village. 
Reste le délicat sujet de la communauté rurale, villageoise ou d'habitants. En effet, la communauté représente d'une certaine façon l'extrême limite de ce qui peut être perceptible par l'archéologie, dans le sens où seules les expressions matérielles de la vie communautaire, en dehors de la chartre l'instituant, peuvent être appréhendées. Il se pose, à ce niveau, un second problème qui est l'identification objective de cette expression matérielle, à l'exclusion de projections fantasmées sous l'influence de la pensée marxiste. Si des éléments comme la place (à bien distinguer de la cour), le cimetière, le réseau viaire, les réseaux fossoyés ou la morphologie agraire peuvent être interprétés comme les traces matérielles d'une vie communautaire, il est plus difficile de se prononcer pour des structures telles que les puits ou les fours, ou encore des espaces comme les zones d'activités spécifiques. À cela s'ajoute que la vie communautaire est parfois distinguée par certains chercheurs de la communauté institutionnalisée.

Se pose la question de la stabilité et du territoire. Il est inutile de revenir sur le critère des matériaux éphémères qui correspond avant tout

9. Il est toujours délicat de donner un chiffre exact. Un nombre supérieur à 5 paraît convenir pour le début du premier Moyen Âge, il doit probablement être revu à la hausse à partir du milieu du VII ${ }^{e}$ siècle.

10. Ce constat est toujour d'actualité: «À mon avis, Cadenet risque de se transformer davantage au cours des quinze ou vingt prochaines années, tant sur le plan agricole que sur le plan de l'habitat, qu'au cours du siècle qui vient de s'écouler", J. Krim, Lettre d'un maire de village, Paris, Seuil, 1971, p. 16, in Le Goff, 2012, p. 167. 11. Il ne s'agit pas ici de remettre en question la thèse avancée sur la spatialisation du social, mais de contester l'idée que des hommes résidan en un même lieu avant le $\mathrm{XI}^{e}$ siècle ne puissent avoir aucun lien social, notamment de voisinage. 12. Qu'ils s'agissent de la thèse portant sur L'Archéologie de l'habitat rural dans la moitié nord de la France (Peytremann 2003 ; 2010), des projets collectifs de recherche L'Habitat rural du haut Moyen Âge en Île-deFrance (Gentili, Lefèvre, 2009), L'Habitat rural au Moyen Âge dans le nordouest de la France (Valais, 2012), L'Habitat rural du Moyen Âge en région Centre, dirigée par A. Nissen et S. Jesset ou de contenu de manuels (Catteddu, 2009; Burnouf et al., 2009).

plusieurs reprises, été précisée par les archéologues qui distinguent sur la longue durée la stabilité d'un terroir et celle des habitats qui en dépendent. Ces derniers étant plus sensibles aux transferts de propriété, aux développements technologiques, notamment en agriculture, aux crises écologiques et anthropiques et in fine aux transformations politiques et religieuses. Les villages de Vallange ou de Yutz en Moselle (Blaising, 200o) sont, à ce titre, exemplaires. La permanence villageoise souvent avancée est relative et en aucun cas un modèle unique. Elle se partage le devenir des villages avec la désertion qui intervient régulièrement et à toute période. Il est en revanche difficile de faire la part des deux, au haut Moyen Âge, en l'absence d'un corpus étayé de villages actuels archéologiquement étudiés.

Pour finir, il est possible d'affirmer que certains sites du premier Moyen Âge correspondent à des villages. Ces derniers présentent comme caractéristiques principales, quelle que soit leur date de création, de regrouper de manière plus ou moins serrée un minimum d'habitations ${ }^{9}$ ou d'unités d'exploitation qui entretiennent entre de chemins, clôtures, etc. Ces villages possèdent généralement, à partir au plus tôt du milieu du $\mathrm{VII}^{\mathrm{e}}$ siècle, une aire funéraire, associée ou non à un bâtiment religieux. Ils sont établis sur un terroir qui leur assure une grande part de leur activité économique. Il est en revanche difficile, sauf dans un raisonnement théorique pur, de concevoir que des habitants de parcelles voisines, parfois séparées par une clôture, n'aient pas de lien social. Il convient néanmoins de rester prudent sur la notion de «définition » du village altomédiéval, dans le sens où celle-ci figerait un objet qui se caractérise avant tout par ses transformations permanentes opérées selon un rythme irrégulier ${ }^{10}$. Le village dans sa matérialité est le témoin des changements sociaux et religieux qui modifient au fil du temps les besoins de ses habitants.

Mais si l'existence du village est assurée au premier Moyen Âge, loin s'en faut pour que l'ensemble des «habitats ruraux du premier Moyen Âge » soit des villages.

Pour une grande partie des sites, la superficie explorée et/ou les conditions de conservation ne permettent pas de mesurer l'importance du groupement des habitations ni de percevoir leur organisation. D'autres sites correspondent à des fermes isolées ou à des groupements insuffisamment importants pour être qualifiés de village. Au demeurant, certains sites sont totalement dépourvus de maisons d'habitation. Cette diversité des modalités de peuplement qui transparaît derrière la diversité des sites est en adéquation avec la pluralité des dénominations des lieux habités que l'on retrouve dans la documentation textuelle. Et c'est là, probablement, une piste pour sortir du village et de ses ornières épistémologiques. elles des liens perceptibles dans l'aménagement

\section{Les problématiques actuelles}

Si les propos de Jacques Morsel sur les habitats ${ }^{11}$ apparaissent provocants ou même choquants pour les archéologues médiévistes, c'est bien parce que, face au débat sur le village, ils se sont finalement accommodés de l'expression «habitat rural» qui présentait l'avantage de venir $\mathrm{du}$ «terrain» et de ne pas susciter d'interrogations, notamment concernant la communauté villageoise. De fait, l'ensemble des découvertes de sites ruraux domestiques réalisées dans le cadre de l'archéologie préventive sont dénommées sans distinction «habitat rural». Le même constat peut être établi au sujet des travaux les concernant $^{12}$. C'est la raison pour laquelle il convient de souligner la parution de l'article intitulé «Histoire(s) de(s) village(s).

L'archéologie en contexte villageois : un enjeu pour la compréhension de la dynamique des habitats médiévaux» (Carré et al., 2009) et celle des actes d'une table ronde, Archéologie du village, archéologie dans le village dans le nord de la France ( $V^{e}$-XIII siècles) (Mahé-Hourlier, Poignant, 2013).

Depuis peu, deux questionnements mettent à mal l'expression «habitat rural». Le premier vient des découvertes de plus en plus nombreuses de sites élitaires qui attirent l'attention sur les critères de distinctions sociales permettant d'identifier une résidence élitaire (Bourgeois, 2006 et 2010 ;

Nissen-Jaubert, 2003 et 2010 ; Peytremann, 2013). Le second plaide pour une ouverture lexicale face à la variété des découvertes archéologiques que l'expression «habitat rural» ne permet pas de recouvrir (Peytremann, à paraître), afin de pouvoir commencer à mener des analyses comparatives

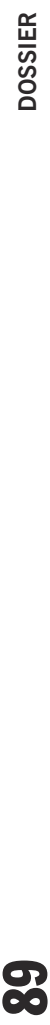

$\mathscr{\infty}$ 
intrasites. Timidement, dans la bibliographie, apparaissent des articles consacrés à des zones de stockage, des zones d'activités spécialisées, des quartiers artisanaux, rarement d'ailleurs les archéologues osent employer le terme de «village». Ces deux axes de recherche indiquent que les archéologues sont actuellement prêts à reposer la question de la nature de ce qu'ils fouillent et à s'interroger sur les fonctions auxquelles les données renvoient, mais aussi à aborder les aspects collectifs (pour ne pas dire communautaires) qu'ils perçoivent. Ils sont aidés en cela par le développement des études paléoenvironnementales et archéogéographiques qui prolongent le lieu de résidence et de travail à l'espace de production agricole et d'exploitation du milieu naturel. C'est là le troisième angle d'approche qui peut permettre de comprendre les réseaux de peuplement, leur inscription dans le terroir et leurs rapports avec d'autres réseaux, comme celui des paroisses, dont le centre est matérialisé par l'église et les contours plus flous (Morsel, 2007, p. 16), ou politique avec celui des lieux de pouvoir.

Le contexte présent visant à protéger les terres agricoles incite les aménageurs à se rapprocher des centres villageois, offrant ainsi la possibilité, tant attendue par les archéologues, d'explorer les sous-sols des villages actuels pour tenter de comprendre non seulement l'origine et la dynamique des habitats, mais aussi leur hiérarchisation au premier Moyen Âge. Il est souhaitable que ce quatrième axe de recherche, particulièrement prometteur puisqu'il apportera des données complémentaires à celles engrangées depuis trente ans, ne soit pas victime de la législation actuelle en matière de zonage de présomption de prescriptions d'interventions archéologiques et du seuil de déclenchement, dans la mesure où les projets portent nécessairement sur des surfaces nettement plus restreintes que ceux développés jusqu'à présent en dehors des villages.

À l'heure où les sociologues parlent de la « fin du village $»^{\mathbf{1 3}}$ pour évoquer la dissolution des liens communautaires, la transformation des fonctions économiques et la modification du bâti, il est important de ne pas accentuer le sentiment nostalgique et de renforcer ainsi l'archétype du village. La forme de peuplement qu'il représente reste pérenne dans son aspect groupé, sa localisation à la campagne et sa proximité avec les espaces funéraires. Les autres fonctions sont étroitement liées aux sociétés et à leurs besoins, et sont donc sujettes à de nombreuses transformations.

Le village altomédiéval représente une étape particulière dans l'histoire du village. Celle du rapprochement des morts et des vivants, de l'apparition progressive d'un lieu de culte chrétien associé aux habitations, d'une rationalisation de l'organisation et de l'exploitation du terroir dans lequel il est ancré. 


\section{Références bibliographiques}

BLAISING J.-M., 2000: «Évolution de l'habitat sur le terroir de Yutz (Moselle) », in KLÁPŠTĚ J. Structures de l'habitat rural médiéval, groupement et dispersion, Actes du congrès Ruralia III Maynooth, 3-9 sept. 1999, Prague, Institute of Archaeology, p. 120-155.

BouRgEOIS L., 2006: «Les résidences des élites et les fortifications du haut Moyen Âge en France et en Belgique dans leur cadre européen : aperçu historiographique (1995-2005)», Cahiers de civilisation médiévale, 49, p. 113-142.

BouRGEOIS L., 2010 (DIR.): Une résidence des comtes d'Angoulême autour de l'an mil. Le Castrum d'Andonne. Fouilles d'André Debord, Caen, Publications du Crahm, $560 \mathrm{p}$

Burnouf J., Arribet-Derouin D., Desachy B. Journot F., NisSen-Jaubert A., 2009: Manue d'archéologie médiévale et moderne, Paris, A. Colin, $384 \mathrm{p}$.

Carré F., Hincker V., Mahé N., Peytremann E. POIGNANT S., ZADORA-RIO É., 2009: «Histoire(s) de(s) village(s). L'archéologie en contexte villageois, un enjeu pour la compréhension de la dynamique des habitats médiévaux", Les Nouvelles de l'archéologie, 116, p. 51-59.

CATteddu I., 1992: « L'habitat rural mérovingien de Genlis (Côte-d'Or) », Revue archéologique de l'Est, 43, 161, p. 39-98.

CAtteddu I., 2009: Archéologie médiévale en France: le premier Moyen Âge ( $V^{e}-X I^{e}$ siècle), Paris La Découverte, $177 \mathrm{p}$

Chapelot J., Fossier R., 1980: Le Village et la maison au Moyen Âge, Paris, Hachette, $357 \mathrm{p}$.

Châtelet M. (DIR.), 2002: Imling «Le Haut des Oies». Le village disparu de Sarrixing (570344 $10 \mathrm{AH}$ ) (Moselle), D.F.S. de sauvetage urgent (10/05/1999 17/o9/1999), Metz, SRA Lorraine, 4 vol. dactylographiés.

CHRISTLEIN R., 1980 : "Kircheim bei München, Oberbayern. Das Dorf des Frühen Mittelalters », Das archäologische Jahr in Bayern, p. 162-163.

Cursente B., 2004: «Village», in Gauvard C., De Libera A., Zink M. (DIR.), Dictionnaire $d u$ Moyen Âge, Paris, Quadrige/PUF, p. 1448-1450.

DANNHEIMER H., 1973: «Die frühmittelaterliche Siedlung bei Kircheim (Ldkr München, Oberbayern). Vorbericht über die Untersuchungen im Jahre 1970 ", Germania, 51, p. 152-169.

Du CANGe C., 1883-1887: «Villagium», in DU CANGE C. et al., Glossarium medice et infimce latinitatis [éd augm.], Niort, L. Favre, t. 8, col. 330c., http://ducange.enc.sorbonne.fr/

Fossier R. 1982: Enfance de l'Europe. Aspects économiques et sociaux. 1/ L'homme et son espace, 2/ Structures et problèmes, Paris, PUF, 2 vol., $1125 \mathrm{p}$.
Fossier R., 1992 : «Villages et villageois », in Villages et villageois au Moyen Âge, Actes du XXXI ${ }^{e}$ Congrès de la Société des historiens médiévistes de l'enseignement supérieur public, Caen,1990, Paris, Publications de la Sorbonne (coll. « Histoire ancienne et médiévale », 26), p. 207-214.

FouRQUIN G., 1975 : «Le premier Moyen Âge », in Duby G. et WAllon A. (DIR.), Histoire de la France rurale, vol. 1, La formation des campagnes françaises des origines au XIV viècle, Paris, Seuil, p. 286-371.

Gauvard C., De Libera A., Zink M. (DIR.), 2004², Dictionnaire du Moyen Âge, Paris, Quadrige/PUF, $1548 \mathrm{p}$.

Gentili F., 2010, «L'organisation spatiale des habitats ruraux du haut Moyen Âge ", in CHAPELOT J. (DIR.), Trente ans d'archéologie médiévale en France: un bilan pour un avenir. Actes du $9^{e}$ Congrès international de la Société d'archéologie médiévale, Vincennes, 16-18 juin 2006, Caen, Publications du Crahm, p. 119-131.

Gentili F., Lefèvre A. (DIR.), 2009: Programme collectif de recherche. L'habitat rural du haut Moyen Âge en Île-de-France. Bilan 2004/2006, Guiry-en-Vexin, Centre de recherches archéologiques du Vexin français (coll. Suppl. au Bulletin archéologique du Vexin français), p. 97-126.

GÉRARD F., 2012: « La structuration du village pour une économie agraire planifiée à la fin du IX ${ }^{\mathrm{e}}$ siècle en Lorraine. Les sites de Vitry-sur-Orne et de Demange-aux-Eaux », Archéopages, 34, p. 38-47.

JESSET S., à paraître: «Les ateliers de potiers du haut Moyen Âge autour d'Orléans: caractérisation, organisation et production ", in Actes du colloque de Douai du 5 au 8 octobre 2010, Tourner autour du pot. Les ateliers de potiers médiévaux $d u v^{e} a u$ $X I I^{e}$ siècle dans l'espace européen.

Le Goff J.-P., 2012: La Fin du village, Paris, Gallimard, $577 \mathrm{p}$.

LORREN C., PÉRIN P. (DIR.), 1995 : L'Habitat rural du haut Moyen Âge (France, Pays-Bas, Danemark et Grande-Bretagne), Actes des XIVe Journées internationales d'archéologie mérovingienne, Guiry-en-Vexin et Paris, 4-8 février 1993, Rouen, Association française d'archéologie mérovingienne (coll. «Mémoires », VI), 237 p.

Mahé-Hourlier N., Poignant S. (ÉD.), 2013: Archéologie du village, Archéologie dans le village dans le nord de la France (Ve-XIII siècles). Actes de la table ronde Archéologie du village, archéologie dans le village dans le nord de la France $V^{e}$-XIII ${ }^{e}$ siècles, Saint-Germain-en-Laye, 22-24 novembre 2007, Saint-Germain-en-Laye, Afam (Mémoires, XXIX), $249 \mathrm{p}$.

Morsel J., 2007: L'Histoire (du Moyen Âge) est un sport de combat... Réflexions sur les finalités de l'histoire du Moyen Âge destinées à une société dans laquelle même les étudiants d'histoire s'interrogent, Paris, LAMOP-Paris 1, 197 p. [En ligne] https://lamop. univ-paris1.fr/IMG/pdf/SportdecombatMac.pdf.

NisSEN-JAUBERT A., 2003: «Historiographie de la spatialisation des élites: les approches archéologiques », in Historiographie des élites dans le haut Moyen Âge. Table ronde du 28 novembre 2003 organisée par le Lamop, [en ligne] http:/ lamop.univ-parisı.fr/lamop/elites/index.htlm.
NisSEN-JAUberT A., 2010: «Late antiquity and early medieval high-status sites in France», in Herrenhöfe und die Hierarchie der Macht im Raum südlich und östlich der Nordsee von der Vorrömischen Eisenzeit bis zum frühen Mittelalter und zur Wikingerzeit - Herrenhöfe and the Hierarchy of Power in the Region to the South and East of the North Sea from the Pre-Roman Iron Age until the Early Middle Ages and the Viking Age. Burg Bederkesa in Bad Bederkesa 11.-13. Oktober 2007, Rahden, M. Leidorf (Siedlungs- und Küstenforschung im südlichen Nordseegebiet, 33), p. 240-252.

PÉRIN P., 1992: « La part du haut Moyen Âge dans la genèse des terroirs de la France médiévale », in Parisse M., Barral I et Altet X. (Dir.), Le roi de France et son royaume autour de l'an mil, actes du colloque Hugues Capet 987-1987. La France de l'an mil, Paris-Senlis, 22-25 juin 1987, Paris, Picard, p. 225-234.

Petit M. (DIR.), 2009: L'Habitat carolingien des Sureaux à la Grande-Paroisse (Seine-et-Marne). Une communauté villageoise à l'aube de l'an Mil, Nemours, Conseil général de Seine-et-Marne (coll. «Mémoires archéologiques de Seine-etMarne»), $199 \mathrm{p}$.

Peytremann É., 2003: Archéologie de l'habitat rural dans le nord de la France du IV $V^{e}$ au XII siècle, Saint-Germain-en-Laye, Association française d'archéologie mérovingienne (coll. «Mémoires », XIII), 2 vol., 452 et $432 \mathrm{p}$.

Peytremann É., 2013: «Identifier les résidences des élites au sein des habitats ruraux $\mathrm{du} \mathrm{VI}^{\mathrm{e}}$ au $\mathrm{XI}^{\mathrm{e}}$ siècle dans la moitié nord de la France ", in KLÁPŠTĚ J. (ED.), Hierarchies in Rural Settlements. Actes du IXe colloque international Ruralia, Götzis, 26 septembre-9 octobre 2011, Turnhout, Brepols Publishers, 2013, p. 183-197.

Peytremann É., à paraître: « Pour une approche renouvelée des habitats ruraux du haut Moyen Âge dans la moitié nord de la France ", in Actes de la table ronde Pratiques de l'espace. Archéologie et histoire des territoires médiévaux, organisée à l'occasion du cinquantenaire du Crahm, Caen, 29 février- $1^{\text {er }}$ mars 2008

RAYNAUD C., 2003: «Le système de peuplement de l'âge du Fer au Moyen Âge », in BATS M., DEDET B., Garmy P., Janin T., Raynaud C., Schwaller M. (DIR.), Peuples et territoires en Gaule méditerranéenne. Hommage à Guy Barruol, Montpellier, Association de la Revue archéologique de Narbonnaise (coll. Suppl. à la $R A N, 35)$, p. 323-354.

STORK I., 1997: « Friedhok und Dorf, Herrenhof und Adelgrab. Der einmalige Befund Laucheim », in ARCHÄOLOGISCHEN LANDESMUSEUM BADEWÜrTtenbERG (ÉD.), Die Alamanen, Stuttgart, Theiss, p. 290-310.

VAlais A. (DIR.), 2012: L'Habitat rural au Moyen Âge dans le nord-ouest de la France, t. 1, Les Synthèses, Rennes, Presses universitaires de Rennes, 328 p.

VERHULST A., 1992: «Village et villageois ", in Villages et villageois au Moyen Âge, Actes du XXXI ${ }^{e}$ Congrès de la Société des historiens médiévistes de l'enseignement supérieur public, Caen, 199o, Paris, Publications de la Sorbonne (coll. Histoire ancienne et médiévale, 26), p. 9-13.

Watteaux M., 2003: «À propos de la "naissance du village du Moyen Âge". La fin d'un paradigme?», Études rurales, 167-168, p. 307-318.

ZADORA-Rio É., 1995: «Le village des historiens et le village des archéologues ", in Campagnes médiévales: l'homme et son espace. Études offertes à Robert Fossier, Paris, Publications de la Sorbonne, p. 145-153.

ZADORA-RIO É., GALINIÉ H., 2003: « La fouille du site de Rigny ( VII $^{\mathrm{e}}$-XIX ${ }^{\mathrm{e}}$ siècles) et la question de la genèse des centres paroissiaux ", Les Nouvelles de l'archéologie, 92, p. 24-27. 